Article

\title{
The Abelian Kernel of an Inverse Semigroup
}

\author{
A. Ballester-Bolinches * (D) and V. Pérez-Calabuig
}

Departament de Matemàtiques, Universitat de València, Dr. Moliner 50, 46100 Burjassot, València, Spain; vpercal@gmail.com

* Correspondence: Adolfo.Ballester@uv.es

Received: 6 July 2020; Accepted: 22 July 2020; Published: 24 July 2020

check for updates

\begin{abstract}
The problem of computing the abelian kernel of a finite semigroup was first solved by Delgado describing an algorithm that decides whether a given element of a finite semigroup $S$ belongs to the abelian kernel. Steinberg extended the result for any variety of abelian groups with decidable membership. In this paper, we used a completely different approach to complete these results by giving an exact description of the abelian kernel of an inverse semigroup. An abelian group that gives this abelian kernel was also constructed.
\end{abstract}

Keywords: finite semigroup; abelian kernels; profinite topologies; partial automorphisms; extension problem

\section{Introduction}

This paper concerns the computability of kernels of finite semigroups with respect to varieties of groups. The kernel of a finite semigroup $S$ with respect to a variety of finite groups $\mathfrak{F}$ is defined to be the set of all elements that relate to 1 under every relational morphism from $S$ to a group from $\mathfrak{F}$. Being able to compute such kernels is the key to computing Mal'cev products of decidable varieties of semigroups with $\mathfrak{F}$. In turn, the computation of these Mal'cev products is intimately related with the computation of semidirect products of varieties of semigroups with $\mathfrak{F}$, which plays an important role in the Krohn-Rhodes decomposition theory of finite semigroups. In the case of the variety of all finite groups, which is the most interesting case from the point of view of the Krohn-Rhodes theory, Rhodes and Tilson [1] showed how to compute the regular elements of the kernel, which led to a conjecture for a structural description of the kernel that became known as the Rhodes "Type II" Conjecture. Ash [2] gave an alternative proof of the theorem of Rhodes and Tilson in which the inverse semigroups play an important role.

There are also other varieties of groups for which the associated kernel is computable: the variety of all finite $p$-groups [3], $p$ a prime, varieties of abelian groups [4,5], or the variety of all finite nilpotent groups [6]; however the problem remains open for the varieties of all finite soluble groups and all finite groups of odd order.

The problem of computing the abelian kernel of a finite semigroup, that is, the kernel associated to the variety $\mathfrak{A} \mathfrak{b}$ of all finite abelian groups, was first solved by Delgado in [4]. Steinberg [5] extended Delgado's result for any variety of finite abelian groups with decidable membership. Both results provide an algorithm which decides whether a given element of a semigroup $S$ belongs to the $\mathfrak{A} \mathfrak{b}$-kernel.

In this paper, we use a completely different approach which allows us to enrich and complete Delgado's theorem. Our main result gives a complete description of the abelian kernel of every finite inverse semigroup. In addition, we also describe how to construct an abelian group and a relational morphism $\tau$ which provides the abelian kernel of a finite inverse semigroup.

The proof our main result depends heavily on the description of the relational morphisms between the members of a class of inverse semigroups and abelian groups (Lemma 6). As a consequence, 
every relational morphism can be defined in terms of the quotient of a free abelian group constructed from an inverse semigroup. We then determine the set of elements of the abelian kernel by means of a normal subgroup of the free abelian group whose quotient is finite. This quotient group is the natural candidate to be the image of a relational morphism which gives the abelian kernel.

This result is closely related to profinite topologies and the problem of extending partial automorphisms, which provides extra motivation for undertaking such problems. In a forthcoming paper [7], we present in detail how our result can be applied in this context.

All semigroups and groups considered in this paper are finite.

Section 2 of the paper is intended to collect the basic results on semigroups and kernels. A certain amount of what is here should be considered folklore, although probably some bits are new. Section 3 develops the fundamental concept of matrix of projections onto a 0 -minimal $\mathcal{J}$-class of an inverse semigroup, and on those foundations is then defined the notion of minimal pairs. A key reduction lemma of the computability of kernels to minimal pairs is then proved. Finally, Section 4 contains the proof of our main result.

\section{Background and Notation}

In this section, we collect some definitions and results which constitute the background of this paper. For further details or undefined notation, see [8] or [9].

\subsection{Basic Results on Semigroups}

Let $S$ be a semigroup. For a subset $X$ of $S$, we use $\mathrm{E}(X)$ for the subset of all idempotents of $S$ contained in $X$ and $\langle X\rangle$ is the subsemigroup generated by $X ; X^{1}$ and $X^{0}$ will denote the sets obtained from $X \subseteq S$ by adjoining an identity element 1 and a zero element 0 , respectively. If $Y$ is a subset of $G$, we write $X Y=\{x y: x \in X, y \in Y\}$ for all $X, Y \subseteq S$. If $X=\{x\}$ or $Y=\{y\}$, we simply write $X Y=x Y$ and $X Y=X y$ respectively.

A subset $I$ of a semigroup $S$ is said to be an ideal of $S$ if $S I S \subseteq I$. In this case, the set $S / I=(S \backslash I)^{0}$ is a semigroup in which products falling in $I$ are zero.

The notion of ideal leads naturally to the consideration of an equivalence relation $\mathcal{J}$ introduced by Green which is defined by the rule $s \mathcal{J} t$ if, and only if, $S^{1} s S^{1}=S^{1} t S^{1}$. We will use $J_{s}$ to denote the $\mathcal{J}$-class of an element $s \in S$. In particular, if $S$ is a semigroup with a zero element, then $J_{0}=\{0\}$.

Since $\mathcal{J}$ is defined in terms of ideals, the inclusion order among these ideals induce a preorder $\leq_{\mathcal{J}}$ among the elements of $S: s \leq_{\mathcal{J}} t$ if, and only if, $S^{1} s S^{1} \subseteq S^{1} t S^{1}$. If $s \leq_{\mathcal{J}} t$ and $S^{1} s S^{1} \neq S^{1} t S^{1}$, we write $s \Varangle \mathcal{J} t$.

The following well-known lemma will be very useful when dealing with $\mathcal{J}$-classes of products.

Lemma 1. Let $s, t \in S$. Then $s t \leq \mathcal{J}$ s and $s t \leq \mathcal{J} t$.

Note that $\leq \mathcal{J}$ induces a partial order among the $\mathcal{J}$-classes of $S$ : if $J=J_{S^{\prime}} J^{\prime}=J_{S^{\prime}}$ are $\mathcal{J}$-classes of $S$ for some $s, s^{\prime} \in S$, then $J \leq \mathcal{J} J^{\prime}$ if, and only if, $s \leq \mathcal{J} s^{\prime}$. If $J \leq \mathcal{J} J^{\prime}$ and $J \neq J^{\prime}$, we write $J \leq_{\mathcal{J}} J^{\prime}$.

In general, we write just $\mathcal{J}$ to denote the relation $\mathcal{J}$ on a semigroup $S$; however, when $\mathcal{J}$ is considered in different semigroups $S$ and $T$, we write $\mathcal{J}_{S}$ and $\mathcal{J}_{T}$ to denote, respectively, the relation $\mathcal{J}$ on each of such semigroups.

Let $S$ be a semigroup with zero. A $\mathcal{J}$-class $J$ of $S$ is called 0 -minimal, if for every $0 \neq s \in S, J_{s} \leq \mathcal{J} J$ implies $J_{s}=J$. In particular, for every $s \in J, S^{1} s S^{1}=J^{0}$. By Lemma $1, J_{s t} \leq \mathcal{J} J_{s}=J_{t}$ for all $s, t \in J$. Hence, $J_{s t}=J_{s}=J_{t}$, for every $s, t \in J$ with $s t \neq 0$. Consequently, $J^{0}$ is a subsemigroup of $S$.

If $e \in \mathrm{E}(S)$, then $e S e$ is the set of all elements of $S$ for which $e$ is a two-sided identity. The group of units of $e S e$ is denoted by $S_{e}$. Clearly $S_{e}$ contains all subgroups of $S$ having $e$ as their identity element. Hence $S_{e}$ is called the maximal subgroup of $S$ with $e$ as the identity element. This subgroup of $S$ is denoted by $H_{e}$ in $[8,9]$ and called the maximal subgroup of $S$ at $e$. Our notation is more convenient when maximal subgroups in different subsemigroups containing the same idempotent $e$ are considered. 
Two ideas of great importance in semigroup theory are those of regular element and an inverse of an element. An element $x$ of a semigroup $S$ is called regular if $x \in x S x$, i.e., $x=x S x$, for some $S \in S$. If $s$ is an element of $S$, we say that $t$ is an inverse of $s$, if $s t s=s$ and $t s t=t$.

If $x$ is regular, there exists $s \in S$ such that $x=x s x$, and then $x(s x s) x=x s x=x,(s x s) x(s x s)=$ $(s x)(s x s)=s x s$; i.e., $s x s$ is an inverse of $x$. Moreover, $x s, s x \in \mathrm{E}(S)$.

A $\mathcal{J}$-class $J$ of a semigroup $S$ is said to be regular if $J=J_{s}$ for some regular element $s \in S$, or equivalently $J=J_{e}$ for some $e \in \mathrm{E}(S)$. A semigroup $S$ is called regular if every element of $S$ is regular, or equivalently, if every element of $S$ has an inverse element.

A regular semigroup $S$ is said to be an inverse semigroup if every element $s \in S$ has a unique inverse, $s^{-1}$ say. If $X$ is a subset of an inverse semigroup $S$ we denote $X^{-1}=\left\{x^{-1}: x \in X\right\}$.

The following lemma is very useful (see [8]).

Lemma 2. 1. A subsemigroup $T$ of an inverse semigroup $S$ is an inverse subsemigroup if a $\in T$ implies $a^{-1} \in T$.

2. If $s, t$ are elements of an inverse semigroup $S$, we have that $\left(s^{-1}\right)^{-1}=s$ and $(s t)^{-1}=t^{-1} s^{-1}$.

3. $S$ is an inverse semigroup if, and only if, $S$ is regular and its idempotents commute. As a consequence, if $S$ is an inverse semigroup, $\mathrm{E}(S)$ is a subsemigroup of $S$.

A semigroup $S$ with zero is called 0 -simple if $S^{2} \neq 0$ and 0 is the only proper ideal of $S$.

An inverse 0-simple semigroup is called a Brandt semigroup.

Let $S$ be an inverse semigroup and $J$ a 0 -minimal $\mathcal{J}$-class of $S$. Then, $J^{0}$ is an inverse subsemigroup of $S$, since $s \in J$ if, and only if, $s^{-1} \in J$. Moreover, 0 is the only proper ideal of $J^{0}$, because $S^{1} s S^{1}=J^{0}$, for every $s \in J$. On the other hand, $\left(J^{0}\right)^{2} \neq 0$ because $J$ is a regular $\mathcal{J}$-class. Hence, $J^{0}$ is a Brandt subsemigroup of $S$. Note that if $e \in \mathrm{E}(J)$, then $J_{s}=J_{e}$, for every $s \in S_{e}$. In particular, $\left(J^{0}\right)_{e}=S_{e}$, for every $e \in \mathrm{E}(J)$.

Let $A, B$ be non-empty finite sets and let $G$ be a group. A Rees matrix $C$ is a map $C: B \times A \longrightarrow G^{0}$. We say that $C$ is regular if every row and every column of $C$ has a non-zero entry.

The Rees matrix semigroup with sandwich matrix $C$ is the semigroup $\mathcal{M}^{0}(G, A, B, C)$ with underlying set $(A \times G \times B) \cup\{0\}$ and the operation: $0 \cdot(a, g, b)=(a, g, b) \cdot 0=0$, and

$$
(a, g, b) \cdot\left(a^{\prime}, g^{\prime}, b^{\prime}\right)= \begin{cases}\left(a, g C\left(b, a^{\prime}\right) g^{\prime}, b^{\prime}\right) & \text { if } C\left(b, a^{\prime}\right) \in G \\ 0, & \text { if } C\left(b, a^{\prime}\right)=0\end{cases}
$$

for all $a, a^{\prime} \in A, b, b^{\prime} \in B, g \in G$.

It is known that the Rees matrix semigroup with sandwich matrix $C$ is a regular semigroup if, and only if, $C$ is a regular matrix, and in this case $\mathcal{M}^{0}(G, A, B, C)$ is a 0 -simple semigroup.

The following theorem due to Rees [10] is fundamental.

Theorem 1. Let $S$ be a 0 -simple semigroup. Then, there exists a group $G$ such that $S_{e}$ is isomorphic to $G$, for all $0 \neq e \in \mathrm{E}(S)$, and $S$ is isomorphic to a regular Rees matrix semigroup $\mathcal{M}^{0}(G, A, B, C)$. Conversely, every regular Rees matrix semigroup $\mathcal{M}^{0}(G, A, B, C)$ is a 0 -simple semigroup.

In particular, inverse 0-simple semigroups are called Brandt semigroups and are isomorphic to Rees matrix semigroups of the form $\mathcal{M}^{0}\left(G, \Lambda, \Lambda, I_{\Lambda}\right)$, where $G$ is a group and $\Lambda$ is a set of indices.

We bring the section to a close with the following:

Construction: Let $J=J_{s}$ be a $\mathcal{J}$-class of a semigroup $S$ with zero. Set $X_{J}:=\left\{t \in S: s \leq_{\mathcal{J}} t\right\}$. By Lemma 1, $S \backslash X_{J}$ is an ideal of $S$. Therefore $S_{J}:=S /\left(S \backslash X_{J}\right)=\left(X_{J}\right)^{0}$ is a semigroup with zero. Note that if $S$ is inverse, then $S_{J}$ is inverse as well. 
Recall that the product in $S_{J}$ is defined by:

$$
x \cdot y= \begin{cases}x y, & \text { if } x y \in X_{J} \\ 0, & \text { otherwise }\end{cases}
$$

Let $0 \neq a, b \in S_{J}$. Assume that $a \leq \mathcal{J}_{S} b$. Then, there exist $x, y \in S^{1}$ such that $a=x b y$. By Lemma 1 , $x, y \in S_{J}^{1}$ and so $a \leq_{\mathcal{J}_{S_{J}}} b$. This shows that relations $\mathcal{J}$ coincide in both semigroups, so that $J$ is the unique 0 -minimal $\mathcal{J}$-class of $S_{J}$.

\subsection{Relational Morphisms and Kernels}

A relational morphism $\tau: S \rightarrow T$ between two semigroups $S$ and $T$ is a map from $S$ into $\mathcal{P}(T)$, the set of subsets of $T$, such that $\tau\left(s_{1}\right) \neq \varnothing$ and $\tau\left(s_{1}\right) \tau\left(s_{2}\right) \subseteq \tau\left(s_{1} s_{2}\right)$, for all $s_{1}, s_{2} \in S$. If $\tau$ is a relational morphism between monoids a third condition is required: $1 \in \tau(1)$. In particular, homomorphisms of semigroups are relational morphisms.

Given $\tau_{1}: S \rightarrow \rightarrow T$ and $\tau_{2}: T \rightarrow \rightarrow U$ two relational morphisms, we can define:

$$
\left(\tau_{2} \circ \tau_{1}\right)(s)=\bigcup\left\{\tau_{2}(t): t \in \tau_{1}(s)\right\}, \text { for every } s \in S .
$$

Then $\tau_{2} \circ \tau_{1}: S \rightarrow \rightarrow U$ is again a relational morphism between $S$ and $U$.

If $\tau: S \rightarrow \rightarrow$ is a relational morphism between a semigroup $S$ and a group $G$, the set $\tau^{-1}(1)=$ $\{s \in S: 1 \in \tau(s)\}$ is a subsemigroup of $S$ called the kernel of $\tau$. If $e \in \mathrm{E}(S), e \in \tau^{-1}(1)$, because $\tau(e)$ is a subgroup of $G$.

Suppose, in addition, that $S$ is an inverse semigroup and let $s \in S$. Then we have that $\tau\left(s^{-1}\right)=$ $(\tau(s))^{-1}$. In fact, take $g \in \tau(s)$ and $h \in \tau\left(s^{-1}\right)$. Since $G$ is finite, there exists $n \in \mathbb{N}$ such that $(g h)^{n}=$ 1. Therefore, $(h g)^{n-1} h=g^{-1}$ and $(h g)^{n-1} h \in\left(\tau\left(s^{-1}\right) \tau(s)\right)^{n-1} \tau\left(s^{-1}\right) \subseteq \tau\left(s^{-1}\right)$. Hence, we have $(\tau(s))^{-1} \subseteq \tau\left(s^{-1}\right)$. Analogously, $\tau\left(s^{-1}\right)^{-1} \subseteq \tau(s)$ and the equality holds.

For the sake of clarity, given an element of a Rees matrix semigroup $(i, g, j) \in \mathcal{M}^{0}(G, A, B, C)$, we denote its image under a relational morphism $\tau(i, g, j)$ instead of $\tau((i, g, j))$.

The following proposition analyses the behavior of the images of a unique 0 -minimal $\mathcal{J}$-class of an inverse semigroup $S$ under relational morphisms.

Proposition 1. Let $S$ be an inverse semigroup with a unique 0 -minimal $\mathcal{J}$-class $J$, such that $J^{0}=$ $\mathcal{M}^{0}\left(G, \Lambda, \Lambda, I_{\Lambda}\right) \leq S$. Let $H$ be a group and $\tau: S \rightarrow H$ be a relational morphism. Then, the following properties hold:

1. For every $i \in \Lambda, \tau(i, 1, i)=: K_{i} \leq H$

2. Given $(i, g, j) \in J$, for every $x \in \tau(i, g, j), \tau(i, g, j)=K_{i} x=x K_{j}$.

3. For every $s, t \in J,|\tau(s)|=|\tau(t)|$ and $\tau(s) \tau(t)=\tau($ st $)$, in case that $s t \neq 0$.

Proof. 1. It follows from the fact that relational morphisms between a semigroup and a group send idempotents to subgroups.

2. Let $(i, g, j) \in J$. Since $S$ is an inverse semigroup we have $\tau\left(j, g^{-1}, i\right)=(\tau(i, g, j))^{-1}$.

Let $x \in \tau(i, g, j)$. Then $(i, 1, i)(i, g, j)=(i, g, j)$ and therefore:

$$
K_{i} x \subseteq \tau(i, 1, i) \tau(i, g, j) \subseteq \tau(i, g, j)
$$

Let $y \in \tau(i, g, j)$. Then, $y x^{-1} \in \tau(i, g, j) \tau\left(j, g^{-1}, i\right) \subseteq \tau(i, 1, i)=K_{i}$, thus $y \in K_{i} x$. We can conclude that $\tau(i, g, j)=K_{i} x$.

Analogously, since $(i, g, j)=(i, g, j)(j, 1, j)$, we can also prove that $x K_{j}=\tau(i, g, j)$.

3. By Statement $2, \tau(i, 1, j)=K_{i} x=x K_{j}$, for every $x \in \tau(i, 1, j)$. Therefore, $K_{i}^{x}=K_{j}$, for every $x \in \tau(i, 1, j)$. 
By 2, $|\tau(s)|=|\tau(t)|$, for all $s, t \in J$. Moreover, if $s t \neq 0$, we have that $|\tau(s)| \leq|\tau(s) \tau(t)| \leq$ $|\tau(s t)|=|\tau(s)|$ and then $|\tau(s) \tau(t)|=|\tau(s t)|$, i.e., $\tau(s) \tau(t)=\tau(s t)$.

From now on, we shall be interested here in relational morphisms between semigroups and groups in a variety.

Recall that a formation is a class of groups $\mathfrak{F}$ which is closed under taking epimorphic images and subdirect products. A variety (or pseudovariety) is a formation which is closed under taking subgroups.

Let $\mathfrak{F}$ be a formation. Each group $G$ has a smallest normal subgroup with quotient in $\mathfrak{F}$; this subgroup is called the $\mathfrak{F}$-residual of $G$ and it is denoted by $G^{\mathfrak{F}}$. Clearly $G^{\mathfrak{F}}$ is a characteristic subgroup of $G$ and it is the intersection of all normal subgroups $N$ of $G$ such that $G / N \in \mathfrak{F}$ (see [11], Section 2 for further details).

Let $\mathfrak{F}$ be a variety of groups. The intersection $\mathrm{K}_{\mathfrak{F}}(S)$ of the kernels of all relational morphisms $\tau: S \rightarrow \rightarrow G$ between $S$ and every group $G \in \mathfrak{F}$ is a subsemigroup of $S$ called the $\mathfrak{F}$-kernel of $S$. Since $S$ is finite, there exists $\tau: S \rightarrow \rightarrow G$ with $G \in \mathfrak{F}$ such that $\tau^{-1}(1)=\mathrm{K}_{\mathfrak{F}}(S)$. Moreover, if $W$ is a subsemigroup of $S$, then $\mathrm{K}_{\mathfrak{F}}(W)$ is a subsemigroup of $\mathrm{K}_{\mathfrak{F}}(S)$. If, in addition, $W$ is a subgroup of $S$, then $W^{\mathfrak{F}}$ is contained in $\mathrm{K}_{\mathfrak{F}}(W)$.

If $\mathfrak{F}$ is a variety, the $\mathfrak{F}$-kernel $\mathrm{K}_{\mathfrak{F}}(S)$ of a semigroup $S$ is computable if, and only if, $\mathrm{K}_{\mathfrak{F}}(S) \cap J$ is computable, for every $\mathcal{J}$-class $J$ of $S$. Hence, in the sequel, we shall be concerned about the computability of $\mathrm{K}_{\mathfrak{F}}(S) \cap J$. In this context, the following theorem of Steinberg [12] is the most optimal result so far.

Theorem 2. Let $\mathfrak{F}$ be a variety of groups. We can compute the regular elements of $\mathrm{K}_{\mathfrak{F}}(S)$ for every semigroup $S$ if, and only if, $\mathrm{K}_{\mathfrak{F}}(\bar{S}) \cap J$ is computable for every inverse semigroup $\bar{S}$ and every $\mathcal{J}$-class $J$ of $\bar{S}$.

The following proposition allows us to conclude that it is enough to consider inverse semigroups with zero with a unique 0 -minimal $\mathcal{J}$-class in order to compute the $\mathfrak{F}$-kernel. It was proved by Rhodes and Tilson in [1] for the variety of all groups, and it still holds for a general variety of groups $\mathfrak{F}$.

Proposition 2 ([1], Fact 2.17). Let I be an ideal of S. Then

$$
\mathrm{K}_{\mathfrak{F}}(S / I) \backslash\{0\}=\mathrm{K}_{\mathfrak{F}}(S) \cap(S \backslash I) .
$$

Lemma 3. Let $S$ be a semigroup with zero and let $J$ be a $\mathcal{J}$-class of $S$. Then

$$
\mathrm{K}_{\mathfrak{F}}(S) \cap J=\mathrm{K}_{\mathfrak{F}}\left(S_{J}\right) \cap J .
$$

Proof. Consider the ideal $S \backslash X_{J}$, where $J=J_{s}$ and $X_{J}=\left\{t \in S: s \leq_{\mathcal{J}} t\right\}$. Then $S_{J}=S /\left(S \backslash X_{J}\right)$. By Proposition 2,

$$
\mathrm{K}_{\mathfrak{F}}\left(S_{J}\right) \backslash\{0\}=\mathrm{K}_{\mathfrak{F}}(S) \cap\left(S \backslash\left(S \backslash X_{J}\right)\right)=\mathrm{K}_{\mathfrak{F}}(S) \cap X_{J} .
$$

In particular, since $J \subseteq X_{J}$, we have that $\mathrm{K}_{\mathfrak{F}}\left(S_{J}\right) \cap J=\mathrm{K}_{\mathfrak{F}}(S) \cap J$.

According to the construction at the end of Section 2.1, $S_{J}$ is a semigroup with zero and $J$ is the unique 0 -minimal $\mathcal{J}$-class of $S_{J}$. Hence we have:

Corollary 1. Let $\mathfrak{F}$ be a variety of groups. Then, $\mathrm{K}_{\mathfrak{F}}(S) \cap J$ is computable for every inverse semigroup $S$ and every $\mathcal{J}$-class $J$ of $S$ if, and only if, $\mathrm{K}_{\mathfrak{F}}(\bar{S}) \cap \bar{J}$ is computable for every inverse semigroup $\bar{S}$ with zero having a unique 0-minimal $\mathcal{J}$-class $\bar{J}$.

Proposition 3. Let $S$ be a semigroup and let $\mathfrak{F}$ be a variety of groups. Then, for every $e \in \mathrm{E}(S),\left(S_{e}\right)^{\mathfrak{F}}$ is a subgroup of $\left(\mathrm{K}_{\mathfrak{F}}(S)\right)_{e}$. 
Proof. Let $e \in \mathrm{E}(S)$. Since $S_{e} \leq S$, we have that $\mathrm{K}_{\mathfrak{F}}\left(S_{e}\right) \leq \mathrm{K}_{\mathfrak{F}}(S)$. Since $\left(S_{e}\right)^{\mathfrak{F}}$ is a subgroup of $\mathrm{K}_{\mathfrak{F}}\left(S_{e}\right)$ and $e$ is the identity element of $\left(S_{e}\right)^{\mathfrak{F}}$, it follows that $\left(S_{e}\right)^{\mathfrak{F}} \leq\left(\mathrm{K}_{\mathfrak{F}}(S)\right)_{e}$.

\section{Minimal Pairs}

Before proving our main result, we must deal with some structural facts concerning inverse semigroups. These will allow us to formalize the notion of minimal pairs on which we reduce the study of the computability of kernels.

Throughout the section, $S$ is an inverse semigroup with zero and $J$ is a 0 -minimal $\mathcal{J}$-class of $S$. In this case, $J^{0}$ is a Brandt subsemigroup of $S$ which is isomorphic to a regular Rees matrix semigroup of the form $\mathcal{M}^{0}\left(G, \Lambda, \Lambda, I_{\Lambda}\right)$, where $S_{e}=\left(J^{0}\right)_{e} \cong G$, for every $e \in \mathrm{E}(J)$ (see [8]). In fact, without loss of generality we can assume that $J^{0}=\mathcal{M}^{0}\left(G, \Lambda, \Lambda, I_{\Lambda}\right)$.

The following lemma is elementary.

Lemma 4. The following statements hold:

1. For every $(i, g, j),\left(i^{\prime}, g^{\prime}, j^{\prime}\right) \in \mathcal{M}^{0}\left(G, \Lambda, \Lambda, I_{\Lambda}\right), 0 \neq(i, g, j)\left(i^{\prime}, g^{\prime}, j^{\prime}\right)$ if, and only if, $j=i^{\prime}$. Moreover, $(i, g, j)^{-1}=\left(j, g^{-1}, i\right)$.

2. $\mathrm{E}\left(J^{0}\right)=\{0\} \cup\{(i, 1, i): i \in \Lambda\}$ and $S_{(i, 1, i)}=\left(J^{0}\right)_{(i, 1, i)}=(i, G, i)$.

3. For every $s \in S$ and $x \in J^{0}, s x, x s \in J^{0}$.

Statement 3 of Lemma 4 is telling us that $S$ acts on $J^{0}$ by left and right multiplication. The following concepts arise naturally from these actions (see [1]).

If $s \in S$, we consider the sets:

$$
\alpha_{s}:=\{i \in \Lambda:(i, 1, i) s \neq 0\}, \quad \omega_{s}:=\{j \in \Lambda: s(j, 1, j) \neq 0\}
$$

Note that if $i \in \alpha_{s}$, then $0 \neq(i, 1, i) s=(i, g, j)$, for some $j \in \Lambda$ and $g \in G$. Therefore, $(i, 1, i) s(j, 1, j)=(i, g, j)(j, 1, j)=(i, g, j)$ and then $j \in \omega_{s}$. Analogously, if $j^{\prime} \in \omega_{s}$, then $0 \neq s\left(j^{\prime}, 1, j^{\prime}\right)=$ $\left(i^{\prime}, g^{\prime}, j^{\prime}\right)=\left(i^{\prime}, 1, i^{\prime}\right) s$, for some $i^{\prime} \in \Lambda$ and $g \in G$, and therefore, $i^{\prime} \in \alpha_{s}$.

Hence, we can define a one-to-one correspondence $l_{s}$ between $\alpha_{s}$ and $\omega_{s}$. For every $i \in \alpha_{s}$, we define $\iota_{s}(i):=j \in \omega_{s}$ if, and only if, $0 \neq(i, 1, i) s=(i, g, j)=s(j, 1, j)$. Moreover, for every $i \in \alpha_{s}$, $\gamma_{s}(i):=g$ if, and only if, $(i, 1, i)=\left(i, g, l_{s}(i)\right)$, define also a map $\gamma_{s}: \alpha_{s} \rightarrow G$.

Now, let $U_{\Lambda}\left(G^{0}\right)$ be the set of $\Lambda \times \Lambda$ matrices which have at most one non-zero entry in each row and column. It is clear that $U_{\Lambda}\left(G^{0}\right)$ is an inverse semigroup having a unique 0 -minimal $\mathcal{J}$-class $\bar{J}$ formed by the matrices with a unique non-zero element. We are going to prove that there exists a homomorphism between $S$ and $U_{\Lambda}\left(G_{0}\right)$.

For every $s \in S$, let us define $\varphi_{S}(s):=M_{s} \in U_{\Lambda}\left(G^{0}\right)$, where

$$
M_{S}(i, j)=\left\{\begin{array}{ll}
\gamma_{s}(i), & \text { if } i \in \alpha_{s} \text { and } j=\iota_{s}(i) \\
0, & \text { otherwise }
\end{array}, \text { for every } i, j \in \Lambda\right.
$$

which is well-defined, because $\iota_{s}$ is a bijection between $\alpha_{s}$ and $\omega_{s}$ and then it implies that the matrix $M_{S}$ only has at most one non-zero element in each row and column. Moreover, note that $\varphi_{S}$ defines a bijection between $J$ and $\bar{J}$, since $\varphi_{S}((i, g, j))=M_{(i, g, j)}$ is the matrix with the unique non-zero element $M_{(i, g, j)}(i, j)=g$.

Proposition 4. $\varphi_{S}$ is a homomorphism of semigroups.

Proof. Let $s, t \in S$. First, we claim the following

$$
\alpha_{s t}=\left\{i \in \alpha_{s}: \iota_{s}(i) \in \alpha_{t}\right\}=\iota_{s}^{-1}\left(\omega_{s} \cap \alpha_{t}\right),
$$


$\iota_{s t}(i)=\iota_{t}\left(\iota_{s}(i)\right)$ and $\gamma_{s t}(i)=\gamma_{s}(i) \gamma_{t}\left(\iota_{s}(i)\right)$, for every $i \in \alpha_{s t}$.

Suppose $i \in \alpha_{\text {st }}$. Then, $(i, 1, i)(s t) \neq 0$ and therefore, $i \in \alpha_{s}$ because $(i, 1, i) s \neq 0$. Thus:

$$
\begin{aligned}
0 \neq(i, 1, i)(s t) & =((i, 1, i) s) t=\left(i, \gamma_{s}(i), \iota_{s}(i)\right) t= \\
& =\left(i, \gamma_{s}(i), \iota_{s}(i)\right)\left(\iota_{s}(i), 1, \iota_{s}(i)\right) t
\end{aligned}
$$

i.e., $\left(\iota_{s}(i), 1, \iota_{s}(i)\right) t \neq 0$ and $\iota_{s}(i) \in \alpha_{t}$. On the other hand, suppose that $\iota_{s}(i) \in \alpha_{t}$, for some $i \in \alpha_{s}$. Then

$$
\begin{aligned}
(i, 1, i)(s t) & =((i, 1, i) s) t=\left(i, \gamma_{s}(i), \iota_{s}(i)\right) t= \\
& =\left(i, \gamma_{s}(i), \iota_{s}(i)\right)\left(\left(\iota_{s}(i), 1, \iota_{s}(i)\right) t\right)= \\
& \left.=\left(i, \gamma_{s}(i), \iota_{s}(i)\right)\left(\iota_{s}(i), \gamma_{t}\left(\iota_{s}(i)\right), \iota_{t}\left(\iota_{s}(i)\right)\right)\right)= \\
& \left.=\left(i, \gamma_{s}(i) \gamma_{t}\left(\iota_{s}(i)\right), \iota_{t}\left(\iota_{s}(i)\right)\right)\right) \neq 0 .
\end{aligned}
$$

Hence, our claim holds.

Now, we show that, for every $s, t \in S, M_{s t}=M_{s} M_{t}$.

Let $(i, j) \in \Lambda \times \Lambda$ and suppose that $M_{s t}(i, j) \neq 0$. By definition, we have that $i \in \alpha_{s t}$ with $M_{s t}(i, j)=\gamma_{s t}(i)$ and $\iota_{s t}(i)=j$. Then, by the first claim, we have that $i \in \alpha_{s}, \iota_{s}(i) \in \alpha_{t}$ and $\gamma_{s t}(i)=\gamma_{s}(i) \gamma_{t}\left(\iota_{s}(i)\right)$ and $\iota_{s t}(i)=\iota_{t}\left(\iota_{s}(i)\right)=j$. Therefore, we have that $M_{s}\left(i, \iota_{s}(i)\right) \neq 0$ and $M_{t}\left(\iota_{s}(i), \iota_{t}\left(\iota_{s}(i)\right)\right)=M_{t}\left(\iota_{s}(i), j\right) \neq 0$ and by definition:

$$
M_{S}\left(i, \iota_{S}(i)\right)=\gamma_{s}(i), \quad M_{t}\left(\iota_{S}(i), \iota_{t}\left(\iota_{S}(i)\right)\right)=\gamma_{t}\left(\iota_{S}(i)\right)
$$

Since $M_{S}\left(i, \iota_{S}(i)\right)$ is the unique non-zero element of the $i$-row of $M_{s}$, we have that

$$
\left[M_{s} M_{t}\right](i, j)=\left[M_{s} M_{t}\right]\left(i, \iota_{t}\left(\iota_{s}(i)\right)\right)=\gamma_{s}(i) \gamma_{t}\left(\iota_{s}(i)\right)=\gamma_{s t}(i)=M_{s t}(i, j)
$$

Suppose that $M_{s t}(i, j)=0$. Then, $i \notin \alpha_{s t}$ and therefore $i \notin \alpha_{s}$, which implies $M_{s}(i, k)=0$, for every $k \in \Lambda$. Thus $M_{S}(i, k) M_{t}(k, j)=0$, for every $k \in \Lambda$, and then $\left[M_{S} M_{t}\right](i, j)=0$.

Hence, we can set the following definition.

Definition 1. Let $S$ be an inverse semigroup with a 0-minimal $\mathcal{J}$-class J isomorphic to $\mathcal{M}^{0}\left(G, \Lambda, \Lambda, I_{\Lambda}\right)$. For each $s \in S$, we say that $M_{s}:=\varphi_{S}(s) \in U_{\Lambda}\left(G^{0}\right)$ is the matrix of projections of s onto $\mathcal{M}^{0}\left(G, \Lambda, \Lambda, I_{\Lambda}\right)$.

For a set of indices $\Lambda$ and a group $G$, note that $U_{\Lambda}\left(G^{0}\right)$ is an inverse semigroup with a unique 0 -minimal class $\bar{J}$ such that $\bar{J}^{0}$ is isomorphic to $\mathcal{M}^{0}\left(G, \Lambda, \Lambda, I_{\Lambda}\right)$. For the sake of notation, $M \in$ $U_{\Lambda}\left(G^{0}\right)$ denotes both a general element of the semigroup as well as its matrix of projections onto $\mathcal{M}^{0}\left(G, \Lambda, \Lambda, I_{\Lambda}\right)$.

We bring the section to a close by introducing minimal pairs and our reduction lemma.

Definition 2. Let $S$ be an inverse semigroup with a 0 -minimal $\mathcal{J}$-class $J$ isomorphic to $\mathcal{M}^{0}\left(G, \Lambda, \Lambda, I_{\Lambda}\right)$. We say that $(S, J)$ is a minimal pair if for every $s, t \in S, M_{s}=M_{t}$ implies $s=t$.

Note that by Proposition 4, we can see minimal pairs as embedded copies of subsemigroups of $U_{\Lambda}\left(G^{0}\right)$ containing $\bar{J}$. In particular, we have that every subsemigroup $\bar{S} \leq U_{\Lambda}\left(G^{0}\right)$ containing $\bar{J}$ satisfies $(\bar{S}, \bar{J})$ is a minimal pair. Then, we can prove the following reduction lemma.

Lemma 5. Let $\mathfrak{F}$ be a variety of groups. Then, for every inverse semigroup $S$ with zero and a unique 0-minimal $\mathcal{J}$-class $J, \mathrm{~K}_{\mathfrak{F}}(S) \cap J$ is computable if, and only if, for every minimal pair $(\bar{S}, \bar{J}), \mathrm{K}_{\mathfrak{F}}(\bar{S}) \cap \bar{J}$ is computable.

Proof. Only the sufficiency of the condition is in doubt. 
Suppose that for every minimal pair $(\bar{S}, \bar{J})$, we can compute $\mathrm{K}_{\mathfrak{F}}(\bar{S}) \cap \bar{J}$. Let $S$ be an inverse semigroup $S$ with zero with a unique 0 -minimal $\mathcal{J}$-class $J$. For the sake of notation, we can assume $J^{0}=\mathcal{M}^{0}\left(G, \Lambda, \Lambda, I_{\Lambda}\right)$ for a certain $G$ and $\Lambda$. Then, we can consider the inverse semigroup $U_{\Lambda}\left(G^{0}\right)$ and $\varphi_{S}: S \rightarrow U_{\Lambda}\left(G^{0}\right)$.

We define $\bar{S}:=\varphi_{S}(S)$, which is a subsemigroup of $U_{\Lambda}\left(G^{0}\right)$ with the unique 0 -minimal $\mathcal{J}$-class $\bar{J}$ so that $\bar{J}^{0}$ is isomorphic to $J^{0}$ by means of $\varphi_{S}$. Then, $(\bar{S}, \bar{J})$ is a minimal pair. We prove:

$$
\mathrm{K}_{\mathfrak{F}}(S) \cap J=\varphi_{S}^{-1}\left(\mathrm{~K}_{\mathfrak{F}}(\bar{S})\right) \cap \bar{J}
$$

Let $s \in \mathrm{K}_{\mathfrak{F}}(S) \cap J$ and let $\bar{\tau}: \bar{S} \rightarrow \rightarrow F$ be a relational morphism with $F \in \mathfrak{F}$. Then, $\tau: S \rightarrow \neg F$, given by $\tau(s)=\bar{\tau}\left(\varphi_{S}(s)\right)$, is a relational morphism because is a composition of relational morphisms. Since $s \in \mathrm{K}_{\mathfrak{F}}(S) \cap J$, we have $1 \in \tau(s)$ and then $1 \in \bar{\tau}\left(\varphi_{S}(s)\right)$. Consequently, $\varphi_{S}(s) \in \mathrm{K}_{\mathfrak{F}}(\bar{S})$ and so $\varphi_{S}(s) \in \mathrm{K}_{\mathfrak{F}}(\bar{S}) \cap \bar{J}$.

Now, let $M_{\left(i_{0}, g_{0}, j_{0}\right)} \in \mathrm{K}_{\mathfrak{F}}(\bar{S}) \cap \bar{J}$. Let us prove that $\left(i_{0}, g_{0}, j_{0}\right) \in \mathrm{K}_{\mathfrak{F}}(S)$. Let $\tau: S \rightarrow \rightarrow F$ be a relational morphism with $F \in \mathfrak{F}$. For every $M \in \bar{S}$, we define $\bar{\tau}(0)=F$ and

$$
\bar{\tau}(M):=\bigcap\{\tau(i, g, j): M(i, j)=g \neq 0\}
$$

In particular, $\bar{\tau}\left(M_{\left(i_{0}, g_{0}, j_{0}\right)}\right)=\tau\left(i_{0}, g_{0}, j_{0}\right)$, since $M_{\left(i_{0}, g_{0}, j_{0}\right)}=g_{0}$ is the unique non-zero element of $M_{\left(i_{0}, g_{0}, j_{0}\right)}$. If we could prove that $\bar{\tau}$ is a relational morphism we would be done, because in such a case, we would conclude $1 \in \bar{\tau}\left(M_{\left(i_{0}, g_{0}, j_{0}\right)}\right)=\tau\left(i_{0}, g_{0}, j_{0}\right)$.

First we see that, for every $M \in \bar{S}, \bar{\tau}(M) \neq \varnothing$. In fact, let $(i, j) \in \Lambda \times \Lambda$ such that $M(i, j)=g \neq 0$. Then $i \in \alpha_{s}$, and $(i, 1, i) s=(i, g, j)$. Since $1 \in \tau(i, 1, i)$, we have that $\tau(s) \in \tau(i, 1, i) \tau(s) \subseteq \tau(i, g, j)$. Therefore, we have that

$$
\tau(s) \subseteq \bigcap\{\tau(i, g, j): M(i, j)=g \neq 0\}=\bar{\tau}(M) .
$$

Moreover, let $M, M^{\prime} \in \bar{S}$ such that $0 \neq M M^{\prime}$ (the case $M M^{\prime}=0$ is trivial). We want to prove that $\bar{\tau}(M) \bar{\tau}\left(M^{\prime}\right) \subseteq \bar{\tau}\left(M M^{\prime}\right)$. Let $x \in \bar{\tau}(M)$ and $y \in \bar{\tau}\left(M^{\prime}\right)$. In order to prove that $x y \in \bar{\tau}\left(M M^{\prime}\right)$, we take $(i, j) \in \Lambda \times \Lambda$ such that $\left(M M^{\prime}\right)(i, j)=g \neq 0$ and we will see that $x y \in \tau(i, g, j)$.

By the product in $U_{\Lambda}\left(G_{0}\right)$, there exist $k \in \Lambda$ and $g_{1}, g_{2} \in G$ such that $M(i, k)=g_{1}, M^{\prime}(k, j)=g_{2}$ and $\left(M M^{\prime}\right)(i, j)=g=M(i, k) M^{\prime}(k, j)$. As a consequence, we have that $(i, g, j)=\left(i, g_{1}, k\right)\left(k, g_{2}, j\right)$ and then, by definition of $\bar{\tau}$ :

$$
x y \in \tau\left(i, g_{1}, k\right) \tau\left(k, g_{2}, j\right) \subseteq \tau\left(i, g_{1} g_{2}, j\right)=\tau(i, g, j)
$$

\section{The Abelian Kernel of an Inverse Semigroup}

In this section, we present our main result, which provides a nice description of the abelian kernel of an inverse semigroup. Throughout the section additive notation is used for abelian groups.

Let $S$ be an inverse semigroup and $J$ a $\mathcal{J}$-class of $S$, such that $J^{0}$ is isomorphic to $\mathcal{M}^{0}\left(G, \Lambda, \Lambda, I_{\Lambda}\right)$. Firstly, Lemma 3 ensures us that

$$
\mathrm{K}_{\mathfrak{A} \mathfrak{b}}(S) \cap J=\mathrm{K}_{\mathfrak{A} \mathfrak{b}}\left(S_{J}\right) \cap J,
$$

where $S_{J}$ is an inverse semigroup with $J$ as a unique 0 -minimal $\mathcal{J}$-class. Then, by Lemma 5 , there exists a minimal pair $(\bar{S}, \bar{J})$ with $\bar{S} \leq U_{\Lambda}\left(G^{0}\right)$ and $\bar{J}$ the 0 -minimal $\mathcal{J}$-class of $U_{\Lambda}\left(G^{0}\right)$ such that

$$
\mathrm{K}_{\mathfrak{A} \mathfrak{b}}\left(S_{J}\right) \cap J=\varphi_{S}^{-1}\left(\mathrm{~K}_{\mathfrak{A} \mathfrak{b}}(\bar{S}) \cap \bar{J}\right) .
$$

Hence, in order to give a description of the abelian kernel of an inverse semigroup, we can focus on the case of minimal pairs $(S, J)$. Then, for the rest of the section, $(S, J)$ denotes a minimal pair and 
for the sake of notation, we can assume without loss of generality that $J^{0}=\mathcal{M}^{0}\left(G, \Lambda, \Lambda, I_{\Lambda}\right)$ for a certain set of indices $\Lambda$ and a group $G$.

The following lemma, which describes the relational morphisms between minimal pairs and abelian groups, is crucial in the proof of our main result.

Lemma 6. Let $\tau: S \rightarrow \rightarrow A$, with $A \in \mathfrak{A} \mathfrak{b}$. Then, the following statements follow:

1. For every $i, j \in \Lambda, \tau(i, 1, i)=\tau(j, 1, j)=: H$ is a subgroup of $A$. Then, for every $(i, g, j) \in J$, $\tau(i, g, j)=x+H$, for each $x \in \tau(i, g, j)$.

2. For every element $s \in S$, the equality $\tau(i, g, j)=\tau\left(i^{\prime}, g^{\prime}, j^{\prime}\right)$ holds for all $M_{s}(i, j)=g \neq 0, M_{s}\left(i^{\prime}, j^{\prime}\right)=$ $g^{\prime} \neq 0$, where $M_{s}$ is the matrix of projections of $s$.

3. There exists a relational morphism $\bar{\tau}: S \rightarrow A / H$ such that $|\bar{\tau}(s)|=1$, for every $0 \neq s$, and $\bar{\tau}^{-1}(H) \cap$ $J=\tau^{-1}(0) \cap J$.

Proof. 1. On one hand, we know that $\tau(1,1,1)=: H \leq A$. Then, according to the Proposition 1, $\tau(i, 1, i)=\tau(j, 1, j)=H$, for every $i, j \in \Lambda$. As a consequence, for every $(i, g, j) \in J, \tau(i, g, j)=x+H$, for each $x \in \tau(i, g, j)$.

2. Now, let $s \in S$ and let $(i, j),\left(i^{\prime}, j^{\prime}\right) \in \Lambda \times \Lambda$ such that $M_{s}(i, j)=g \neq 0$ and $M_{S}\left(i^{\prime}, j^{\prime}\right)=g^{\prime} \neq 0$. By the proof of Lemma 5, we know that $\tau(s) \subseteq \tau(i, g, j) \cap \tau\left(i^{\prime}, g^{\prime}, j^{\prime}\right)$. But then, we get that $\varnothing \neq$ $\tau(s) \subseteq(x+H) \cap(y+H)$, where $x \in \tau(i, g, j)$ and $y \in \tau\left(i^{\prime}, g^{\prime}, j^{\prime}\right)$. Hence, $x+H=y+H$ and then $\tau(i, g, j)=\tau\left(i^{\prime}, g^{\prime}, j^{\prime}\right)$.

3. Let $0 \neq s \in S$. By the previous statements, we know that there exists $x_{s} \in A$ such that $\tau(i, g, j)=x_{s}+H$, for every $M_{s}(i, j)=g \neq 0$. Then, we can define $\bar{\tau}(s):=x_{s}+H \in A / H$ and $\bar{\tau}(0)=A / H$. Therefore, for every $0 \neq s,|\bar{\tau}(s)|=1$ and clearly, $\bar{\tau}^{-1}(H) \cap J=\tau^{-1}(0) \cap J$.

Now, let us check that $\bar{\tau}$ is a relational morphism. Suppose that $0 \neq s, t \in S$ are such that $s t \neq 0$ (the case $s t=0$ is trivial). Let $(i, j) \in \Lambda \times \Lambda$ such that $M_{s t}(i, j)=g \neq 0$. By the Proposition 4 and the product in $U_{\Lambda}\left(G_{0}\right)$, there exist $k \in \Lambda$ and $g_{1}, g_{2} \in G$ such that $M_{s}(i, k)=g_{1}, M_{t}(k, j)=g_{2}$ and $M_{s t}(i, j)=M_{s}(i, k) M_{t}(k, j)$.

As a consequence, we have that $(i, g, j)=\left(i, g_{1}, k\right)\left(k, g_{2}, j\right)$ and then, by definition of $\bar{\tau}$ :

$$
\begin{aligned}
\bar{\tau}(s t)= & x_{s t}+H=\bar{\tau}\left(i, g_{,}, j\right)=\bar{\tau}\left(\left(i, g_{1}, k\right)\left(k, g_{2}, j\right)\right) \supseteq \\
& \supseteq \bar{\tau}\left(i, g_{1}, k\right)+\bar{\tau}\left(k, g_{2}, j\right)=\bar{\tau}(s)+\bar{\tau}(t)
\end{aligned}
$$

As a consequence, for every minimal pair $(S, J)$, there exists a relational morphism $\tau: S \rightarrow \rightarrow A \in \mathfrak{A b}$ such that $\tau^{-1}(0) \cap J=\mathrm{K}_{\mathfrak{A} \mathfrak{b}}(S) \cap J$ and for every $0 \neq s \in S,|\tau(s)|=1$ and $\tau(s)=\tau(i, g, j)$, for each non-zero entry $M_{s}(i, j)=g$ of the matrix of projections $M_{s}$. In particular, for every $s, t \in S$ with $0 \neq s t$ the equality $\tau(s t)=\tau(s)+\tau(t)$ holds.

Our main result can be now proved.

Theorem 3. Let $(S, J)$ be a minimal pair with $J^{0}=\mathcal{M}^{0}\left(G, \Lambda, \Lambda, I_{\Lambda}\right)$. Let $X=\left\{x_{i}: i \in \Lambda\right\}$ be an alphabet and the infinite abelian group $F_{a b, X} \oplus G / G^{\prime}$, where $F_{a b, X}$ is the free abelian group over $X$ and $G^{\prime}$ is the derived subgroup of $G$. Then

$$
\mathrm{K}_{\mathfrak{A b}}(S) \cap J=\left\{(i, g, j):\left(-x_{i}+x_{j}, g G^{\prime}\right) \in N\right\},
$$

where $N=\left\langle N_{s}: 0 \neq s \in S\right\rangle \unlhd F_{a b, X} \oplus G / G^{\prime}$ with

$$
N_{s}:=\left\{\left(-x_{i}+x_{j}, g_{1} G^{\prime}\right)-\left(-x_{i^{\prime}}+x_{j^{\prime}}, g_{2} G^{\prime}\right): M_{s}(i, j)=g_{1}, M_{s}\left(i^{\prime}, j^{\prime}\right)=g_{2}\right\},
$$

for every $0 \neq s \in S$. 
Proof. Without loss of generality we can suppose that $\Lambda=\{0,1, \ldots, n\}$ for a certain $n \in \mathbb{N}$. Then, $X=$ $\left\{x_{i}: 0 \leq i \leq n\right\}$ and $A:=F_{a b, X} \oplus G / G^{\prime}$ is a finitely generated abelian group.

For each $0 \neq s \in S \backslash J$, according to the matrix of projections $M_{s}$ of $s$, we set

$$
N_{s}:=\left\{\left(-x_{i}+x_{j}, g_{1} G^{\prime}\right)-\left(-x_{i^{\prime}}+x_{j^{\prime}}, g_{2} G^{\prime}\right): M_{s}(i, j)=g_{1}, M_{s}\left(i^{\prime}, j^{\prime}\right)=g_{2}\right\}
$$

and consider the normal subgroup $N:=\left\langle N_{s}: 0 \neq s \in S\right\rangle \unlhd A$. Then, $A / N$ is a finitely generated abelian group and therefore, by the fundamental theorem of finitely generated abelian groups, we can write

$$
\bar{A}:=A / N=\left(\left\langle y_{1}+N\right\rangle \oplus \cdots \oplus\left\langle y_{m}+N\right\rangle\right) \oplus\left(\left\langle z_{1}+N\right\rangle \oplus \cdots \oplus\left\langle z_{m^{\prime}}+N\right\rangle\right),
$$

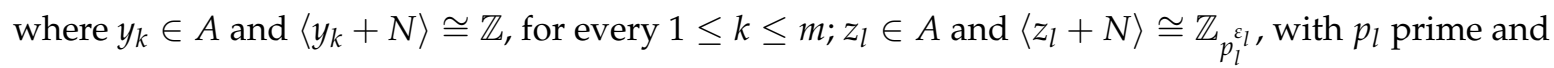
$\varepsilon_{l} \in \mathbb{N}$, for every $1 \leq l \leq m^{\prime}$.

As a consequence, for every $(i, g, j) \in J$, we can write

$$
\left(-x_{i}+x_{j}, g G^{\prime}\right)+N=\left(b_{1} y_{1}+\ldots+b_{m} y_{m}+b_{1}^{\prime} z_{1}+\ldots+b_{m^{\prime}}^{\prime} z_{m^{\prime}}\right)+N,
$$

with $b_{1}, \ldots, b_{m}, b_{1}^{\prime}, \ldots, b_{m^{\prime}}^{\prime} \in \mathbb{Z}$. Write $\varepsilon_{(i, g, j)}:=\left|b_{1}\right|+\ldots+\left|b_{n}\right|$ and $n_{0}:=\max \left\{\varepsilon_{(i, g, j)}:(i, g, j) \in J\right\}$. Consider $\bar{N}:=\left\langle n_{0} y_{1}, \ldots, n_{0} y_{m}\right\rangle \oplus N \unlhd A$. Then

$$
B:=A / \bar{N}=\left(\left\langle y_{1}+\bar{N}\right\rangle \oplus \cdots \oplus\left\langle y_{m}+\bar{N}\right\rangle\right) \oplus\left(\left\langle z_{1}+\bar{N}\right\rangle \oplus \cdots \oplus\left\langle z_{m^{\prime}}+\bar{N}\right\rangle\right) .
$$

Note that $\left\langle y_{k}+\bar{N}\right\rangle \cong \mathbb{Z}_{n_{0}}$, for every $1 \leq k \leq n$, and $\left\langle z_{l}+\bar{N}\right\rangle \cong \mathbb{Z}_{p_{l}^{\varepsilon_{l}}}$, for every $1 \leq l \leq m$. In particular, $B \in \mathfrak{A} \mathfrak{b}$ is a finite abelian group.

Moreover, by the choice of $n_{0}$, it follows that for every $(i, g, j) \in J,\left(-x_{i}+x_{j}, g G^{\prime}\right) \in N$ if, and only if, $\left(-x_{i}+x_{j}, g G^{\prime}\right) \in \bar{N}$. Next we prove that $\mathrm{K}_{\mathfrak{A b}}(S) \cap J=\left\{(i, g, j):\left(-x_{i}+x_{j}, g G^{\prime}\right) \in N\right\}$.

Let $0 \neq s \in S$. Then, for some $(i, j) \in \Lambda \times \Lambda, M_{s}(i, j)=g \neq 0$. Let us define

$$
\tau(s):=\left(-x_{i}+x_{j}, g G^{\prime}\right)+\bar{N} \in B
$$

Note that if $\left(i^{\prime}, j^{\prime}\right) \in \Lambda \times \Lambda$ is such that $M_{s}\left(i^{\prime}, j^{\prime}\right)=g_{2}$ then

$$
\left(-x_{i}+x_{j}, g G^{\prime}\right)+\bar{N}=\left(-x_{i^{\prime}}+x_{j^{\prime}}, g_{2} G^{\prime}\right)+\bar{N} .
$$

Therefore, $\tau$ is well defined. Write $\tau(0)=B$.

Now, let us see that $\tau: S \rightarrow \rightarrow B$ is a relational morphism. Suppose that $0 \neq s t$, for some $s, t \in S$ (the case $s t=0$ is trivial) and let $(i, j) \in \Lambda \times \Lambda$ such that $M_{s t}(i, j)=g \neq 0$. Then, by Proposition 4 and according to the product in $U_{\Lambda}\left(G^{0}\right)$, there exist $k \in \Lambda$ and $g_{1}, g_{2} \in G$ such that $M_{s}(i, k)=g_{1}$, $M_{t}(k, j)=g_{2}$ and $M_{s t}(i, j)=M_{s}(i, k) M_{t}(k, j)$. As a consequence, by definition of $\tau$ :

$$
\begin{aligned}
\tau(s t) & =\left(-x_{i}+x_{j}, g G^{\prime}\right)+\bar{N}=\left(-x_{i}+x_{k}-x_{k}+x_{j},\left(g_{1} g_{2}\right) G^{\prime}\right)+\bar{N} \\
& =\left(-x_{i}+x_{k}, g_{1} G^{\prime}\right)+\bar{N}+\left(-x_{k}+x_{j}, g_{2} G^{\prime}\right)+\bar{N}=\tau(s)+\tau(t)
\end{aligned}
$$

On the other hand, we know that there exists a relational morphism $\bar{\tau}: S \rightarrow \circ \bar{B} \in \mathfrak{A} \mathfrak{b}$ such that $\bar{\tau}^{-1}(0) \cap J=\mathrm{K}_{\mathfrak{A} \mathfrak{b}}(S) \cap J$. According to Lemma 6, for every $0 \neq s \in S$, we can suppose that $\bar{\tau}(s) \in \bar{B}$ and $\bar{\tau}(s)=\bar{\tau}(i, g, j)$, for each non-zero entry $M_{s}(i, j)=g$ of the matrix of projections.

Then, we can define a map $f: X \rightarrow \bar{B}$ given by $f\left(x_{0}\right)=0$ and $f\left(x_{i}\right)=\bar{\tau}(0,0, i) \in \bar{B}$, for every $1 \leq$ $i \leq n$. Therefore, by the universal property of free abelian groups, there exists a unique homomorphism $\bar{f}: F_{a b, X} \rightarrow \bar{B}$, which extends $f$. 
Moreover, recall that by Proposition 3, for every $i \in \Lambda$ we have that $\left(i, G^{\prime}, i\right) \subseteq \mathrm{K}_{\mathfrak{A} \mathfrak{b}}(S) \cap$ $S_{(i, 1, i)}=(i, G, i)$. Then, given $g \in G$, we have that for every $x \in G^{\prime}, 0 \in \bar{\tau}(i, x, i)$ and therefore $\bar{\tau}(i, g x, i)=\bar{\tau}(i, g, i)$.

Hence, we can also consider the map $\delta: G / G^{\prime} \rightarrow \bar{B}$ given by $\delta\left(g G^{\prime}\right)=\bar{\tau}(0, g, 0) \in \bar{B}$, which is well defined by the above mentioned. Moreover, it is a homomorphism, since $\bar{\tau}(0, g, 0)+\bar{\tau}\left(0, g^{\prime}, 0\right)=$ $\bar{\tau}\left(0, g+g^{\prime}, 0\right)$.

Let $\phi: A \rightarrow \bar{B}$ the homomorphism defined by $\phi(w, g)=\bar{f}(w)+\delta\left(g G^{\prime}\right)$, for every $\left(w, g G^{\prime}\right) \in A$. Therefore, $A / \operatorname{ker} \phi \cong \phi(A) \leq \bar{B} \in \mathfrak{A} \mathfrak{b}$. Recall that for every $0 \neq s \in S, \bar{\tau}(s)=\bar{\tau}(i, g, j)$, for each non-zero entry $M_{\mathcal{S}}(i, j)=g$. Moreover

$$
\begin{aligned}
\bar{\tau}(i, g, j) & =\bar{\tau}((i, 0,0)(0, g, 0)(0,0, j))=\bar{\tau}(i, 0,0)+\bar{\tau}(0, g, 0)+\bar{\tau}(0,0, j)= \\
& =-\bar{f}\left(x_{i}+x_{j}\right)+\delta\left(g G^{\prime}\right)=\phi\left(-x_{i}+x_{j}, g G^{\prime}\right) \in \phi(A),
\end{aligned}
$$

i.e., $\bar{\tau}(s) \in \phi(A) \leq \bar{B}$, for every $0 \neq s \in S$. In addition, note that $0=\bar{\tau}(i, g, j)$ if, and only if, $\left(-x_{i}+x_{j}, g G^{\prime}\right) \in \operatorname{ker} \phi$. Therefore, $\bar{\tau}^{-1}(0) \cap J=\left\{(i, g, j):\left(-x_{i}+x_{j}, g G^{\prime}\right) \in \operatorname{ker} \phi\right\}$.

Now, let $0 \neq s \in S$ and suppose that $M_{s}(i, j)=g_{1}$ and $M_{s}\left(i^{\prime}, j^{\prime}\right)=g_{2}$ are non-zero entries of $M_{S}$. Then, by definition of $\bar{\tau}, \bar{\tau}(i, g, j)=\bar{\tau}\left(i^{\prime}, g^{\prime}, j^{\prime}\right)$ and according to the previous paragraph, $\phi\left(-x_{i}+x_{j}, g_{1} G^{\prime}\right)=\phi\left(-x_{i^{\prime}}+x_{j^{\prime}}, g_{2} G^{\prime}\right)$. Thus, $\left(-x_{i}+x_{j}, g_{1} G^{\prime}\right)-\left(-x_{i^{\prime}}+x_{j^{\prime}}, g_{2} G^{\prime}\right) \in \operatorname{ker} \phi$. Therefore, $N_{s} \subseteq \operatorname{ker} \phi$, for every $0 \neq s \in S$, so that $N \leq \operatorname{ker} \phi$.

Hence, we can conclude that

$$
\begin{aligned}
\left\{(i, g, j):\left(-x_{i}+x_{j}, g\right) \in N\right\} & \subseteq\left\{(i, g, j):\left(-x_{i}+x_{j}, g\right) \in \operatorname{ker} \phi\right\}= \\
& =\bar{\tau}^{-1}(1) \cap J=\mathrm{K}_{\mathfrak{A} \mathfrak{b}}(S) \cap J,
\end{aligned}
$$

and the other inclusion is proved.

\section{Conclusions}

Let $S$ be an inverse semigroup with a 0 -minimal $\mathcal{J}$-class $J$ such that $J$ is isomorphic to $\mathcal{M}^{0}\left(G, \Lambda, \Lambda, I_{\Lambda}\right)$, for a set $\Lambda$. Then

$$
\mathrm{K}_{\mathfrak{A} \mathfrak{b}}(S) \cap J=\left\{(i, g, j):\left(-x_{i}+x_{j}, g G^{\prime}\right) \in N\right\}
$$

where $N=\left\langle N_{s}: 0 \neq s \in S\right\rangle \unlhd F_{a b, X} \oplus G / G^{\prime}$ with

$$
N_{s}:=\left\{\left(-x_{i}+x_{j}, g_{1} G^{\prime}\right)-\left(-x_{i^{\prime}}+x_{j^{\prime}}, g_{2} G^{\prime}\right): M_{s}(i, j)=g_{1}, M_{s}\left(i^{\prime}, j^{\prime}\right)=g_{2}\right\}
$$

for every $0 \neq s \in S$, provides a description of the abelian kernel in terms of the infinite abelian group $A:=F_{a b, X} \oplus G / G^{\prime}$. In fact, there exists a finite abelian quotient of $A$ giving the abelian kernel.

Lemma 6 and the proof of Theorem 3 characterize relational morphisms between minimal pairs and abelian groups. In the work in preparation [7], we apply these results to obtain some outcomes within the framework of profinite topologies and partial automorphisms, such as the computation of the pro-abelian closure of a finitely generated subgroup of a free group.

Author Contributions: All authors have contributed equally in the tasks of conceptualization, methodology, validation, investigation and writing-original draft preparation. All authors have read and agreed to the published version of the manuscript.

Funding: This research was funded by Ministerio de Ciencia, Innovación y Universidades and the Agencia Estatal de Investigación, Spain, and FEDER, European Union, grant number PGC2018-095140-B-I00 and Prometeo/2017/057 of Generalitat (Valencian Community, Spain), grant number /2017/057.

Conflicts of Interest: The authors declare no conflict of interest. 


\section{References}

1. Rhodes, J.; Tilson, B.R. Improved lower bounds for the complexity of finite semigroups. J. Pure Appl. Algebra 1972, 2, 13-71. [CrossRef]

2. Ash, C.J. Inevitable graphs: A proof of the type II conjecture and some related decision procedures. Int. J. Algebra Comput. 1991, 1, 127-146. [CrossRef]

3. Ribes, L.; Zalesskiǐ, P.A. The pro-p topology of a free group and algorithmic problems in semigroups. Int. J. Algebra Comput. 1994, 4, 359-374. [CrossRef]

4. Delgado, M. Abelian pointlikes of a semigroup. Semigroup Forum 1998, 56, 339-361. [CrossRef]

5. Steinberg, B. Monoid kernels and profinite topologies on the free Abelian group. Bull. Austral. Math. Soc. 1999, 60, 391-402. [CrossRef]

6. Almeida, J.; Shahzamanian, M.H.; Steinberg, B. The pro-nilpotent group topology on a free group. J. Algebra 2017, 480, 332-345. [CrossRef]

7. Ballester-Bolinches, A.; Pérez-Calabuig, V. Abelian kernels, profinite topologies and partial automorphisms. Unpublished work.

8. Clifford, A.H.; Preston, G.B. The Algebraic Theory of Semigroups; American Mathematical Society: Providence, RI, USA, 1961; Volume I.

9. Rhodes, J.; Steinberg, B. The q-Theory of Finite Semigroups; Springer Monographs in Mathematics; Springer: New York, NY, USA, 2009.

10. Rees, D. On semi-groups. Math. Proc. Camb. Philos. Soc. 1940, 36, 387-400. [CrossRef]

11. Ballester-Bolinches, A.; Ezquerro, L.M. Classes of Finite Groups; Mathematics and Its Applications; Springer: New York, NY, USA, 2006; Volume 584.

12. Steinberg, B. Inevitable graphs and profinite topologies: some solutions to algorithmic problems in monoid and automata theory, stemming from group theory. Int. J. Algebra Comput. 2001, 11, 25-71. [CrossRef]

(C) 2020 by the authors. Licensee MDPI, Basel, Switzerland. This article is an open access article distributed under the terms and conditions of the Creative Commons Attribution (CC BY) license (http:/ / creativecommons.org/licenses/by/4.0/). 